\title{
Inhibition of melanogenesis by Aster yomena callus pellet extract in melanoma cells and patients with skin pigmentation
}

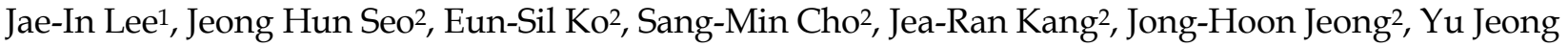 \\ Jeong ${ }^{1}$, Cha Young Kim ${ }^{1}$, Jeong-Dan Cha ${ }^{2}$, Woo Sik Kim ${ }^{1}{ }^{* \bowtie}$, and Young-Bae Ryu ${ }^{1, * \varpi}$ \\ 1. Functional Biomaterial Research Center, Korea Research Institute of Bioscience and Biotechnology, Jeongeup-si, Jeollabuk-do 56212, Republic of Korea \\ 2. Department of Bio-material and product development and R\&D center, General Bio, Namwon-si, Jeollabuk-do 55793, Republic of Korea \\ * The authors contributed equally to this work.
}

$\triangle$ Corresponding author: Dr. Woo Sik Kim, Functional Biomaterial Research Center, Korea Research Institute of Bioscience and Biotechnology, Jeongeup-si, Jeollabuk-do 56212, Republic of Korea, Tel +82-63-570-5171, Email: kws6144@kribb.re.kr or Dr. Young-Bae Ryu, Functional Biomaterial Research Center, Korea Research Institute of Bioscience and Biotechnology, Jeongeup-si, Jeollabuk-do 56212, Republic of Korea, Tel +82-63-570-5171, Email: ybryu@kribb.re.kr

(c) The author(s). This is an open access article distributed under the terms of the Creative Commons Attribution License (https://creativecommons.org/licenses/by/4.0/). See http://ivyspring.com/terms for full terms and conditions.

Received: 2021.05.10; Accepted: 2021.07.05; Published: 2021.07.23

\begin{abstract}
Plant tissue culture holds immense potential for the production of secondary metabolites with various physiological functions. We recently established a plant tissue culture system capable of producing secondary metabolites from Aster yomena. This study aimed to uncover the mechanisms underlying the potential therapeutic effects of Aster yomena callus pellet extract (AYC-P-E) on photoaging-induced skin pigmentation. Excessive melanogenesis was induced in B16F10 melanoma cells using a-melanocyte stimulating hormone ( $\alpha-M S H)$. The effects of AYC-P-E treatment on melanin biosynthesis inducers and melanin synthesis inhibition were assessed. Based on the results, a clinical study was conducted in subjects with skin pigmentation. AYC-P-E inhibited melanogenesis in $\alpha$-MSH-treated B16F10 cells, accompanied by decreased $\mathrm{mRNA}$ and protein expression of melanin biosynthesis inducers, including cyclic AMP response element-binding protein (CREB), tyrosinase, microphthalmia-associated transcription factor (MITF), tyrosinase related protein-1 (TRP-1), and TRP-2. This anti-melanogenic effect was mediated by mitogen-activated protein kinase (MEK)/extracellular signal-regulated kinase (ERK) and protein kinase B (AKT) phosphorylation. Treatment of subjects with skin pigmentation with AYC-P-E-containing cream formulations resulted in 3.33\%, 7.06\%, and $8.68 \%$ improvement in the melanin levels at 2, 4, and 8 weeks, respectively. Our findings suggest that AYC-P-E inhibits excessive melanogenesis by activating MEK/ERK and AKT signaling, potentiating its cosmetic applications in hyperpigmentation treatment.
\end{abstract}

Key words: Aster yomena; callus; extract; metabolite; melanogenesis; skin pigmentation

\section{Introduction}

Nature offers incredibly diverse natural substances that are capable of exerting various physiological effects [1]. Such natural compounds extracted from plants, animals, or other organisms are widely used as ingredients in personal care products and natural cosmetics [2-4]. However, the composition of these substances is greatly affected by environmental factors. For instance, the growth conditions, such as climate or soil parameters, harvest timing, production methods, and storage conditions affect the constitution of natural compounds obtained from plants [5]. Moreover, various environmental pollutants (water quality, air, and soil pollution) can result in mutations and organism extinction [6]. These phenomena present a significant challenge to the homogeneous extraction of natural substances from a variety of biological species that is essential to the cosmetic industry.

In recent years, several studies have actively utilized natural materials possessing excellent 
physiological activity in functional cosmetics using plant tissue culture technology [7-10]. Plant tissue culture is a sterile culture technique that extracts organs, tissues, and cells from a plant, followed by culturing in medium with nutrients in order to regenerate a plant with complete functionality or isolate groups of single cells from a callus [11]. This technique can be used to effectively mass-produce plant cells and organs in a consistent manner through the maintenance, preservation, and periodic subculture of specific cell lines [11-13]. In particular, metabolites (including alkaloids, flavonoids, polyphenols, terpenes, triterpenes, cardiac glycosides, paclitaxel and related taxoids) with various physiologically active functions can be produced using this method $[14,15]$. Therefore, the plant tissue culture technique is actively studied to continuously improve the production of biologically valuable high-quality materials [16-18]. Nonetheless, research on the selection and function of plant callus materials that can be used as functional cosmetic ingredients is still lacking.

Aster yomena, a perennial herb found in the southern regions of South Korea, is widely used as a functional food ingredient in these regions. Recent studies have shown that extracts isolated from this plant using various methods possess various physiological properties, such as anti-obesity, anti-arthritic, and anti-inflammatory properties [19-21]. We have recently succeeded in establishing an Aster yomena callus culture system and confirmed that metabolites with various functions can be produced using this system. Importantly, the metabolite composition of Aster yomena callus pellet extract (AYC-P-E) was found to alleviate skin photoaging [22]. Based on these findings, AYC-P-E is expected to be widely used as a functional cosmetic ingredient capable of preventing skin photoaging. The purpose of this study was to investigate the effects of AYC-P-E on photoaging-induced skin pigmentation. As skin pigmentation is caused by an excessive increase in the melanin content of skin tissues, we first confirmed the anti-whitening effects of AYC-P-E in B16F10 melanoma cells following the induction of excessive melanogenesis using melanin stimulating hormone (a-MSH). Furthermore, based on the in vitro findings, we conducted a clinical study of a cosmetic formulation that contains AYC-P-E in 22 healthy subjects with facial hyperpigmentation.

\section{Materials and methods}

\subsection{Preparation of AYC-P-E}

AYC-P-E was prepared according to previously described protocols [22]. Briefly, sterilized Aster yomena roots were placed into Murashige and Skoog (MS) solid medium supplemented with $1 \mathrm{mg} / 1$ 2,4-dichlorophenoxyacetic acid (2,4-D), 3\% (w/v) sucrose, and $0.4 \%(\mathrm{w} / \mathrm{v})$ Gelrit for 2 weeks $\left(\right.$ at $25^{\circ} \mathrm{C}$ in the dark) to induce callus formation. The resultant cultured callus was inoculated into a $500 \mathrm{ml}$ flask containing $100 \mathrm{ml}$ of a liquid MS1D medium and cultured at $25^{\circ} \mathrm{C}$ in the dark on a rotary shaker at 80 rpm. Next, $100 \mathrm{ml}$ cell suspensions for the exponential phase were transferred to a 21 tank of a sterilized glass bioreactor, and the volume was adjusted to 1.51 with the liquid MS1D medium. The bioreactor was maintained at $25^{\circ} \mathrm{C}$ in the dark, and the callus was incubated for 10 days. After 10 days, the pellets were collected following centrifugation at $12,000 \mathrm{rpm}$ for 30 $\min$ at $4^{\circ} \mathrm{C}$. The collected pellets $(5 \mathrm{~g})$ were suspended in 11 sterile distilled water (DW) and incubated at $80^{\circ} \mathrm{C}$ for $2 \mathrm{~h}$, and the supernatants were collected following centrifugation at $12,000 \mathrm{rpm}$ for $30 \mathrm{~min}$ at $4^{\circ} \mathrm{C}$. The collected solutions were dried in a vacuum freeze drier, and the freeze-dried powders were resuspended in PBS and filtered through a $0.22 \mu \mathrm{m}$ filter. The final concentration of AYC-P-E was adjusted to $30 \mathrm{mg} / \mathrm{ml}$.

\subsection{Melanoma cell line culture}

A cryopreserved B16F10 murine melanoma cell line was obtained from Korea Cell Line Bank (KCLB, Seoul, South Korea). B16F10 cells were grown in the presence of Dulbecco's Modified Eagle's Medium (Gibco BRL, Grand Island, NY, USA) supplemented with 10\% fetal bovine serum (Gibco BRL) and 1\% penicillin/streptomycin (Gibco BRL) at $37^{\circ} \mathrm{C}$ in a humidified incubator $\left(5 \% \mathrm{CO}_{2}\right.$ condition; Thermo Scientific, Waltham, MA, USA).

\subsection{Cell viability and cytotoxicity}

B16F10 cells at passage 5 were used for all the experiments. To investigate the cytotoxic effect of AYC-P-E in melanoma cells, B16F10 cells were seeded in a 6-well plate at a density of $1 \times 10^{5}$ cells/well for 12 $h$, and the media were removed. Then, culture medium containing AYC-P-E at various concentrations $(15,30,60$, and $120 \mu \mathrm{l} / \mathrm{ml})$ was added. After $72 \mathrm{~h}$, cells were harvested and washed twice with phosphate-buffered saline (PBS). The washed cells were stained with Annexin V (diluted 1:50 in Annexin V binding buffer; BD Bioscience, San Diego, CA, USA) and propidium iodide (PI, diluted 1:25 in Annexin $\mathrm{V}$ binding buffer; BD Bioscience) for $15 \mathrm{~min}$ at room temperature $\left(20^{\circ} \mathrm{C} \pm 5\right)$ in the dark and then washed with Annexin V binding buffer. Data were acquired using a Life Launch Attune Nxt Flow Cytometer (ThermoFisher Scientific) and analyzed using the FlowJo software (Version 10; Tree Star, Inc., 
Ashland, OR). Next, to investigate the protective effect of AYC-P-E on melanoma cells against staurosporine (STS)-induced apoptotic damage. B16F10 cells $\left(1 \times 10^{5}\right.$ cells/well $)$ were seeded in a 6 -well plate for $12 \mathrm{~h}$. Then, culture medium containing AYC-P-E (120 $\mu \mathrm{l} / \mathrm{ml})$ and STS (50 nM; Sigma-Aldrich, St. Louis, Mo, USA) was added. After $72 \mathrm{~h}$, cell viability and apoptotic cell death were analyzed using the EZ-Cytox kit (DoGen, Seoul, Korea) and annexin V/PI staining, respectively. Cell viability assay using EZ-Cytox kit was conducted according to the manufacturer's instructions.

\subsection{Melanin content measurement}

B16F10 cells were seeded in a 6-well plate at a density of $5 \times 10^{4}$ cells/well for $12 \mathrm{~h}$, and the media were removed. Then, culture medium (phenol red-free) containing a-MSH (200 nM; Sigma-Aldrich) was added for $1 \mathrm{~h}$ prior to treatment with AYC-P-E for an additional $72 \mathrm{~h}$. To determine the extracellular melanin content, culture supernatants were analyzed at $405 \mathrm{~nm}$ using a microplate reader (Molecular Devices Inc., San Jose, CA, USA). To determine the intracellular melanin content, the cultured cells were lysed using RIPA buffer (Pierce, Rockford, IL, USA) and centrifuged at $12,000 \times \mathrm{g}$ for $1 \mathrm{~h}$ at $4^{\circ} \mathrm{C}$. The collected pellets were then dissolved in $1 \mathrm{M} \mathrm{NaOH}$ containing $10 \% \mathrm{DMSO}$ at $80^{\circ} \mathrm{C}$ for $1 \mathrm{~h}$. The absorbance of the heated solution was analyzed at $405 \mathrm{~nm}$ using a microplate reader.

\subsection{Quantitative real-time polymerase chain reaction (qRT-PCR)}

Culture medium containing a-MSH $(200 \mathrm{nM})$ was added to B16F10 cells $\left(1 \times 10^{5}\right.$ cells/well in a 6-well plate) for $1 \mathrm{~h}$ prior to treatment with AYC-P-E. After $24 \mathrm{~h}$ of AYC-P-E treatment, the cells were harvested, washed twice with cold PBS, and total RNA isolation and qRT-PCR were performed as previously described [22]. Briefly, total RNA was extracted using the easy-spin Total RNA Extraction Kit (iNtRON Biotechnology, Seongnam, South Korea). Only RNA samples with a purity of $260 / 280 \mathrm{~nm}$ ratio 1.8 or higher were used in the experiment using Nanodrop (Maestrogen, Ramsey, MN, USA). cDNA was synthesized using $2 \mu \mathrm{g}$ RNA and a high-capacity cDNA synthesis kit (Bioneer, Daejon, South Korea). Next, to measure the mRNA expression levels, the SYBR Green Mastermix (Applied Biosystems, Roche, USA) and the relevant primers were used to perform qRT-PCR using the Applied Biosystems 7500 Real-Time PCR instrument (Thermo Fisher Scientific). The following primers pairs were used for the qRT-PCR: Microphthalmia-associated transcription factor

(MITF)
5'-CTGTACTCTGAGCAGCAGGTG-3' and reverse, 5'- CCCGTCTCTGGAAACTTGATCG-3'; Tyrosinase forward, 5'-ATAACAGCTCCCACCAGTGC-3' and reverse, 5'-CCCAGAAGCCAATGCACCTA-3'; Tyrosinase related protein-1 (TRP-1) forward, 5'-AGACGCTGCACTGCTGGTCAAGCCTGTAGCC CACGTCGTA-3' and reverse, 5'-GCTGCAGGAGCCTTCTTTCT-3'; Tyrosinase related protein-2 (TRP-2) forward, 5'-GCTCCA AGTGGCTGTAGA-3' and reverse, 5'-AATGCAGTGGCTTGGAAATC-3'; and $\beta$-actin forward, 5'-GCACCACACCTTCTACAATG-3'. The thermal cycling protocol consisted of a hot start $(40$ cycles of $95^{\circ} \mathrm{C}$ for $5 \mathrm{~min}, 95^{\circ} \mathrm{C}$ for $20 \mathrm{sec}, 60^{\circ} \mathrm{C}$ for 20 sec, and $72^{\circ} \mathrm{C}$ for $30 \mathrm{sec}$ ), and a melting curve (at $95^{\circ} \mathrm{C}$ for $1 \mathrm{~min}, 55^{\circ} \mathrm{C}$ for $1 \mathrm{~min}$, and $30^{\circ} \mathrm{C}$ for $1 \mathrm{~min}$ ). The relative expression of each gene was determined by normalizing its expression levels to those of $\beta$-actin.

\subsection{Immunoblotting analysis}

Culture medium containing a-MSH $(200 \mathrm{nM})$ was added to B16F10 cells $\left(1 \times 10^{5}\right.$ cells/well in a 6-well plate) for $1 \mathrm{~h}$ prior to treatment with AYC-P-E. After $24 \mathrm{~h}$ of AYC-P-E treatment, the cells were harvested, and washed twice with cold PBS. The washed cells were lysed using RIPA buffer, and proteins $(20 \mu \mathrm{g})$ were subjected to $10 \%$ SDS-PAGE, and western blotting was performed as previously described [23]. Cyclic AMP response element-binding protein (CREB), phosphorylated (p)-CREB (Ser ${ }^{133}$ ), tyrosinase, microphthalamia-associated transcription factor (MITF), tyrosinase related protein-1 (TRP-1), TRP-2, mitogen-activated protein kinase $1 / 2$ (MEK1/2), p-MEK1/2 (Ser $218 /$ Ser $\left.^{222}\right), \quad$ extracellular signal-regulated kinase 1/2 (ERK1/2), p-ERK1/2 $\left(\mathrm{Thr}^{202} / \mathrm{Tyr}^{204}\right)$, protein kinase B (PKB, also known as AKT), p-AKT (Ser $\left.{ }^{473}\right)$, and $\beta$-actin antibodies used were purchased from Santa Cruz Biotechnology (Dallas, TX, USA).

\subsection{Clinical research}

Clinical research was conducted by the Korea Dermatology Research Institute (KDRI; Bundang, South Korea) for eight weeks. Patients with skin hyperpigmentation on the face (22 female subjects aged 37 to 55 years with an average age of 49 years with phototype III or IV skin according to the Fitzpatrick phototype scale [Fitzpatrick, 1975]) were included in the study. The exclusion criteria were as follows: pregnant or lactating women and women who may be pregnant, patients with a history of photoallergies or photosensitization, those who had been using steroid-containing skin external preparations for more than 1 month, those who had participated in a similar study within 6 months of the 
start of the current study, those with sensitive and irritable skin, those with skin abnormalities, such as spots, acne, erythema, and expansion of capillaries, those who used the same or similar cosmetics or drugs on the same skin area within three months of the beginning of the study, and those who consumed medicine or food claimed to have skin-whitening effects. The clinical research was approved (KDRI-IRB-20275) by the Institutional Review Board (IRB) of the Korea Dermatology Research Institute, and this study was conducted at Seongnam, South Korea, in July to September 2020 in accordance with the WHO guidelines for good clinical practice (GCP) for trials on pharmaceutical products. All protocols were initiated after receiving written consent in compliance with the principles of the Helsinki Declaration.

The test samples were prepared in the form of a cream. In particular, the test sample contained $68.6 \%$ AYC-P-E, and the control group sample did not contain AYC-P-E. The samples were provided in the same container to ensure the double-blind design of the study. The test and control samples were randomly assigned to the left and right sides of the face of each subject, and the allocation was not disclosed to the subjects until the completion of the clinical research. The subjects applied each sample to the designated side of the face twice a day for 8 weeks. At each visit, all subjects washed the tested skin area and entered the constant temperature and humidity chamber $\left(20-24^{\circ} \mathrm{C}, 40-60 \% \mathrm{RH}\right)$ to rest for $30 \mathrm{~min}$ before participating in the test. The evaluation was conducted by measuring the melanin index (M-index) at the site of skin hyperpigmentation at each time point $(2,4$, and 8 weeks) before and after using the sample. The melanin index was defined as the average value obtained by measuring the skin hyperpigmentation five times using a Mexameter ${ }^{\circledR}$ MX 18 (Courage+ Khazaka electronic, $\mathrm{GmbH}$, Germany).

\subsection{Statistical analysis}

The in vitro data are expressed as the mean \pm standard deviation (SD) of at least three independent experiments. The levels of significance for comparisons between samples were determined using one-way analysis of variance (ANOVA) followed by Tukey's multiple comparison test using the GraphPad statistical software (GraphPad Software, San Diego, CA). The clinical study data were analyzed using the Statistical Package for the Social Sciences (SPSS; IBM, USA) program. All data are expressed as the mean \pm standard error of measurement (SEM). Normality and prior homogeneity for data analysis were verified using paired t-tests. The interdependence of the same subject, comparison between groups, and confirmation of changes before and after the indicated time points were analyzed using repeated-measures analysis of variance (RM ANOVA). A P-value of $<0.05$ was considered statistically significant.

\section{Results}

\subsection{AYC-P-E decreases the intracelluar and extracelluar melanin contents in a-MSH-treated murine B 16F 10 melanoma cells}

Our group recently showed the effectiveness of AYC-P-E in improving various phenomena, such as skin damage, inflammatory reaction, decreased type I procollagen, and increased elastase, accompanied by photoaging in UVB-irradiated keratinocytes [22]. The present study aimed to evaluate the anti-melanogenic effects of the 19 metabolites present in AYC-P-E (Fig. 1) and the potential use of AYC-P-E in cosmeceutics as an anti-hyperpigmentation compound. Prior to conducting the clinical study of AYC-P-E in the treatment of hyperpigmentation, we first focused on determining the cellular toxicity of AYC-P-E in murine B16F10 melanoma cells. A low toxicity may offer new insight into the design and development of cosmeceutical ingredients. AYC-P-E cytotoxicity was analyzed by performing Annexin $\mathrm{V}$ and PI staining (Fig. 2A). We found that AYC-P-E is not cytotoxic as there were no remarkable differences in the percentage of dead cells (Annexin $\mathrm{V}^{+} / \mathrm{PI}^{+}$; late apoptotic cells, Annexin $\mathrm{V}^{+} / \mathrm{PI}$; early apoptotic cells, or Annexin $\mathrm{V}^{-} / \mathrm{PI}^{+}$cells; necrotic cells) in B16F10 cultures exposed to AYC-P-E (15, 30, 60, and 120 $\mu \mathrm{l} / \mathrm{ml}$ ) for prolonged periods of time $(72 \mathrm{~h})$. We also confirmed the cytoprotective effect of AYC-P-E on the apoptosis inducer STS-treated melanoma cells (Fig. 2B and C). Interestingly, the B16F10 melanoma cells that were co-treated with AYC-P-E $(120 \mu \mathrm{l} / \mathrm{ml})$ and STS $(50 \mathrm{nM})$ showed significantly reduced percentage of dead cells (late apoptotic cells, early apoptotic cells, and necrotic cells) compared with the STS alone-treated cells (Fig. 2B). Furthermore, STS-treated B16F10 melanoma cells significantly increased cell viability when treated with AYC-P-E (Fig. 2C), indicating that it does have a recovery effect against cellular damages of melanoma cells. Next, to investigate the effect of AYC-P-E on the regulation of melanogenesis in a-MSH-treated murine B16F10 melanoma cells, B16F10 cells were pretreated with a-MSH $(200 \mathrm{nM})$ for $1 \mathrm{~h}$ prior to AYC-P-E treatment $(15,30,60$, and $120 \mu \mathrm{l} / \mathrm{ml}$, for $72 \mathrm{~h})$. Subsequently, we measured the intracellular and extracellular melanin contents. As shown in Fig. 2D, AYC-P-E decreased the intracellular and extracellular melanin contents 
induced by a-MSH stimulation in B16F10 cells in a dose-dependent manner. These results indicate that
AYC-P-E can suppress the synthesis of melanin in a-MSH-stimulated B16F10 cells.
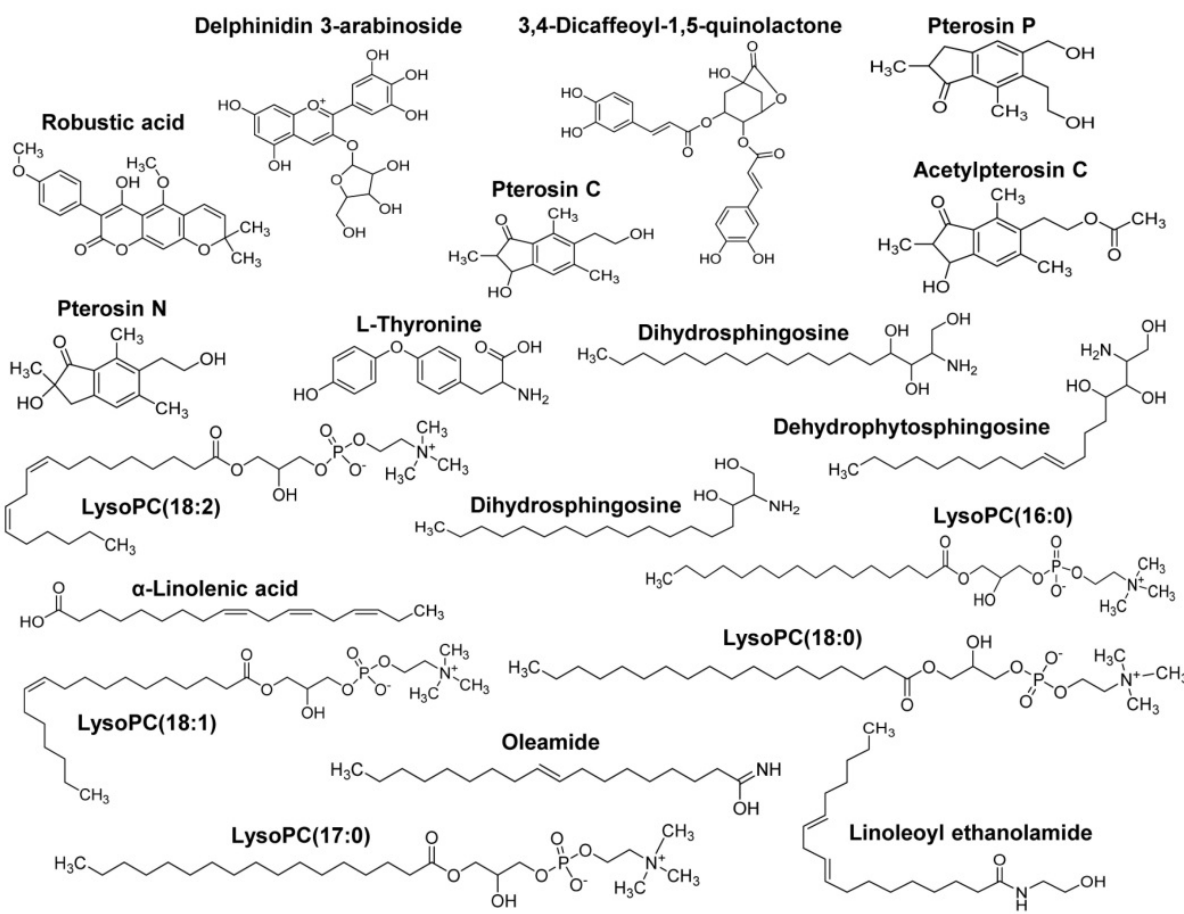

Dehydrophytosphingosine

$$
\mathrm{H}
$$

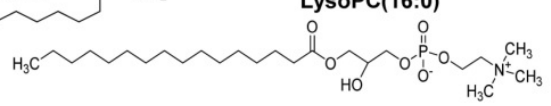

LysoPC(18:0)
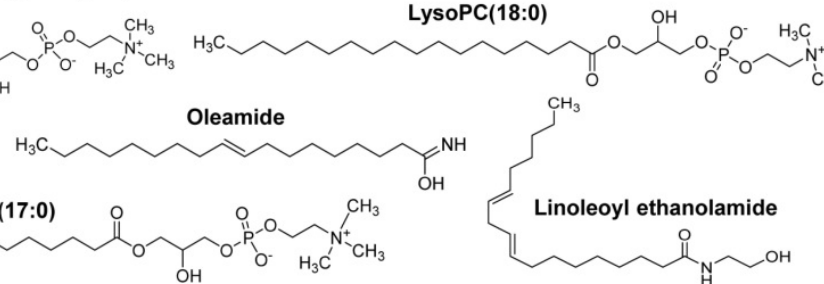

Figure 1. Metabolite composition of AYC-P-E. AYC-P-E includes 19 major metabolites, including one isoflavonoid (robustic acid), one flavonoid (delphinidin 3-arabinoside), four indanes (pterosin C, pterosin P, acetylpterosin C, and pterosin N), 1 cinnamic acid and derivative (3,4-Dicaffeoyl-1,5-quinolactone), one amino acid (L-Thyronine), three sphingolipids (dehydrophytoshingosine, dihydrosphingosine, and phytosphingosine), three fatty amides ( $\alpha$-Linolenic acid, linoleoyl ethanolamide, and oleamide), and five glycerophospholipids (LysoPC(18:2), LysoPC(16:0), LysoPC(18:1), LysoPC(17:0), and LysoPC(18:0)).
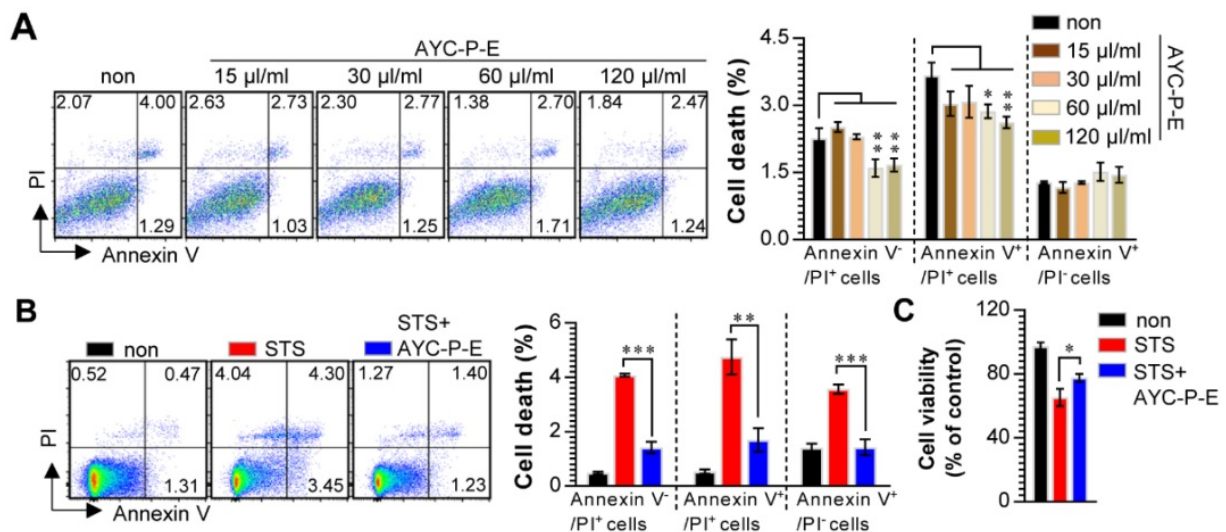

D
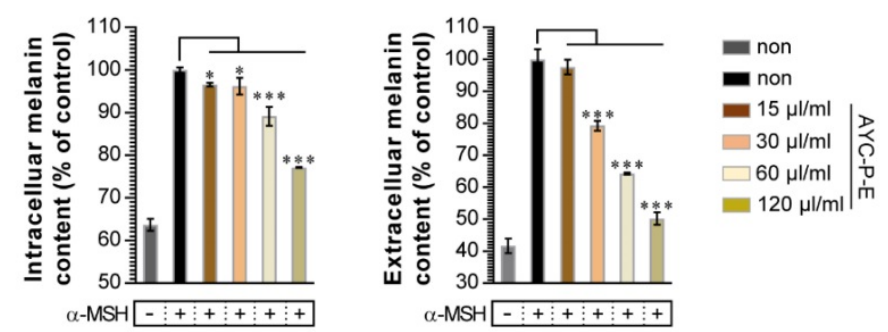

Figure 2. Effect of AYC-P-E treatment on melanin synthesis in $\alpha-M S H$-treated B 16 F1 10 cells. (A and B) B16F10 cells were incubated with culture medium containing AYC-P-E at various concentrations $(15,30,60$, and $120 \mu \mathrm{l} / \mathrm{ml}$ ) in the absence or presence of staurosporine (STS; $50 \mathrm{nM}$ ). After $72 \mathrm{~h}$, cell viability and cell death (necrotic or apoptotic cell death) were measured using EZ-Cytox kit and Annexin V/PI staining, respectively. (A; left panel) The representative plot for Annexin $\mathrm{V}^{+} / \mathrm{PI}+$, Annexin $\mathrm{V}^{+} / \mathrm{PI}$; or Annexin $\mathrm{V}-/ \mathrm{PI}^{+} \mathrm{B} 16 \mathrm{~F} 10$ cells in $\mathrm{AYC}-\mathrm{P}-\mathrm{E}$ alone-treated condition. (A; right panel) The percentages of Annexin $\mathrm{V}^{+} / \mathrm{PI}^{+}, \mathrm{Annexin}^{+} / \mathrm{PI}$, or $\mathrm{Annexin} \mathrm{V} / \mathrm{PI} \mathrm{I}^{+} \mathrm{B} 16 \mathrm{~F} 10$ cells in $\mathrm{AYC}-\mathrm{P}-\mathrm{E}$ alone-treated condition. (B; left panel) The representative plot for Annexin $\mathrm{V}^{+} / \mathrm{PI}^{+}$, Annexin $\mathrm{V}^{+} / \mathrm{PI}$, or Annexin $\mathrm{V}-/ \mathrm{PI}{ }^{+} \mathrm{B} 16 \mathrm{~F} 10$ cells in co-treatment condition with $\mathrm{AYC}-\mathrm{P}-\mathrm{E}$ (120 $\mu \mathrm{l} / \mathrm{ml}$ ) and STS $(50 \mathrm{nM})$. (B; right panel) The percentages of Annexin $\mathrm{V}^{+} / \mathrm{PI}+$, Annexin $\mathrm{V}^{+} / \mathrm{Pl}$-, or Annexin $\mathrm{V}-/ \mathrm{Pl}{ }^{+} \mathrm{B} 16 \mathrm{~F} 10$ cells. (C) Cell viability in co-treatment condition with AYC-P-E $(120 \mu \mathrm{l} / \mathrm{ml})$ and STS $(50 \mathrm{nM})$ was measured using EZ-Cytox kit. (D) The intracellular and extracellular melanin contents of B16F10 cells incubated with culture medium (phenol red-free) containing $\alpha-M S H(200 \mathrm{nM})$ for $1 \mathrm{~h}$ and treated with the indicated concentrations of AYC-P-E for $72 \mathrm{~h}$. All bar graphs show the means \pm standard deviation (SD) of three samples. One representative plot out of three independent experiments is shown; ${ }^{*} \mathrm{P}<0.05,{ }^{*} \mathrm{P}<0.01$, or ${ }^{* * * *} \mathrm{P}<0.001$. 


\subsection{AYC-P-E downregulates the MRNA and protein expression of various factors related to melanogenesis.}

Melanogenesis is mediated by the activation of various signal transduction pathways, such as CREB, tyrosinase, MITF, TRP-1, and TRP-2 [24]. Here, CREB phosphorylation can lead to the activation of MITF expression, which is a transcription factor that plays a critical role in melanocyte development and differentiation. As a result, MITF binds to the promoter regions of the melanin production gene, leading thereby to stimulation (activation of tyrosinase, TRP-1, and TRP-2) of melanogenesis [25]. Therefore, we evaluated whether AYC-P-E treatment inhibits the expression levels of p-CREB, MITF, TRP-1, and TRP-2 induced by a-MSH stimulation in B16F10 cells. As expected, treatment of a-MSH-stimulated B16F10 cells with AYC-P-E significantly inhibited the mRNA (Fig. 3A) and protein (Fig. $3 B$ and $C$ ) expression levels of $\mathrm{p}-\mathrm{CREB}, \mathrm{MITF}$, TRP-1, and TRP-2 compared to those of B16F10 cells treated with a-MSH alone. These results strongly suggest that AYC-P-E can exert anti-melanogenic effects by inhibiting the a-MSH-activated CREB, MITF, TRP-1, and TRP-2 signals in a-MSH-treated B16F10 melanoma cells.

\subsection{AYC-P-E decreases melanogenesis through activation of ERK and AKT signaling.}

Activation of MEK/ERK and AKT signaling phosphorylates MITF, resulting in melanogenesis inhibition. For example, p-MEK-mediated ERK activation (an increase of p-ERK expression) phosphorylates MITF at serine 73, which is followed by MITF ubiquitination and proteasome-mediated degradation [26]. In addition, phosphorylation of AKT can decreases MITF expression via the inhibition of glycogen synthase kinase 3 beta, which downregulates melanogenesis [27]. To examine whether these signals are involved in the anti-melanogenic effects following treatment with AYC-P-E, the activated (phosphorylated) MEK, ERK, and AKT signals were anlayzed using western blot analysis (Fig. 4A and B). We found that a-MSH-stimulated B16F10 cells exhibited significantly enhanced MEK, ERK, and AKT phosphorylation when treated with AYC-P-E compared to that of B16F10 cells treated with a-MSH alone. Next, to confirm the involvement of MEK/ERK and AKT signaling in the AYC-P-E-induced anti-melanogenic effect, we treated a-MSH-stimulated B16F10 cells with specific pharmacological inhibitors (PD98059 for MEK/ERK, LY294002 for AKT) and AYC-P-E and evaluated the intracelluar melanin contents following AYC-P-E treatment (Fig. 4C). These pharmacological inhibitors significantly abrogated the AYC-P-E-induced anti-melanogenic effect (decreased intracelluar melanin content) in a-MSH-stimulated B16F10 cells. Taken together, AYC-P-E was found to activate MEK/ERK and AKT signaling, which led to the suppression of melanin production.

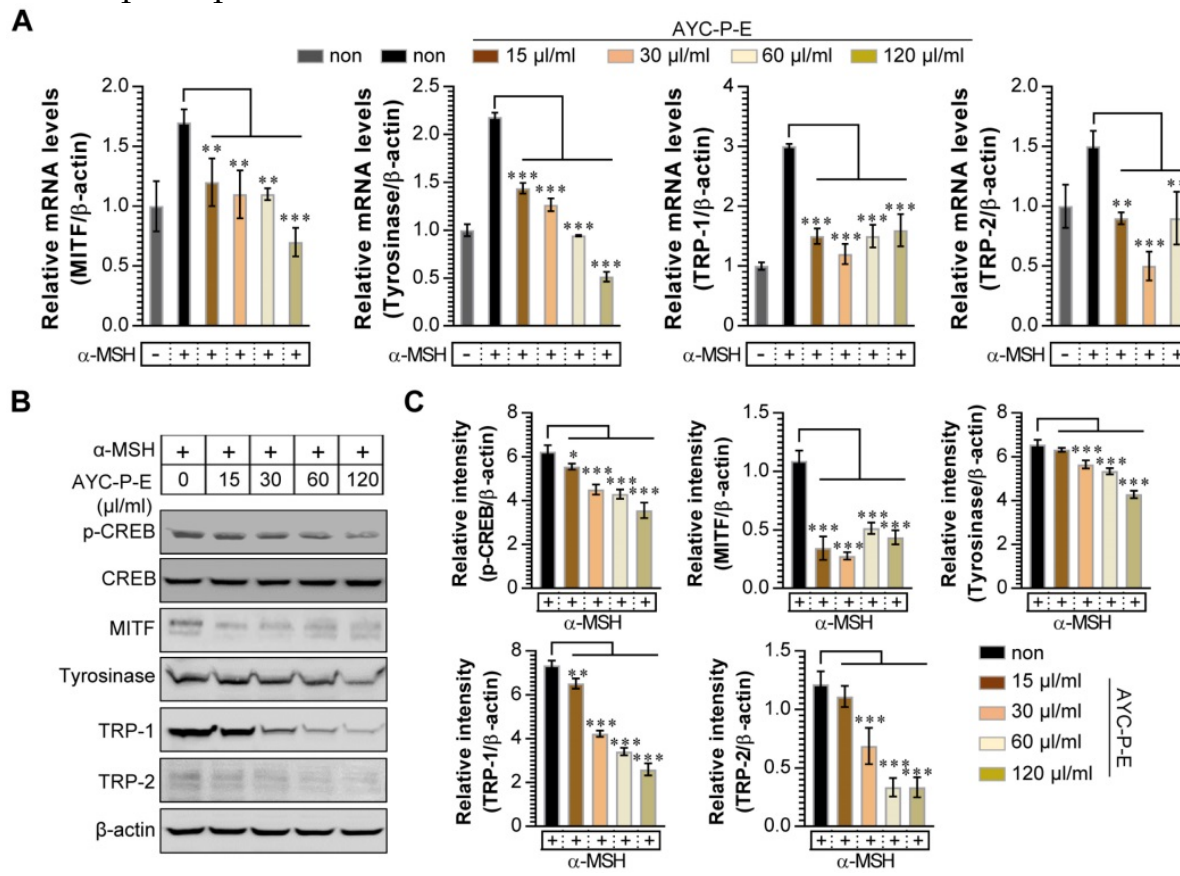

Figure 3. Effect of AYC-P-E treatment on melanogenic protein and mRNA expression in $\alpha$-MSH-stimulated B16F10 cells. (A and B) B16F10 cells were incubated with culture medium containing a-MSH $(200 \mathrm{nM})$ for $1 \mathrm{~h}$ and treated with the indicated concentrations of AYC-P-E for $24 \mathrm{~h}$. (A) The mRNA expression of MITF, tyrosinase, TRP-1, and TRP-2 was analyzed using real-time PCR. (B) Immunoblot analyses were performed using Abs specific to P-CREB (Serl33), MITF, tyrosinase, TRP-1, and TRP-2. CREB and $\beta$-actin was used as the protein loading control. (C) The relative intensity of each protein level is expressed as the mean in the bar graphs. All bar graphs show the means \pm standard deviation $(\mathrm{SD})$ of three samples. One representative plot out of three independent experiments is shown; ${ }^{* *} \mathrm{P}<0.01$, or ${ }^{* * * *} \mathrm{P}<0.001$. 
A

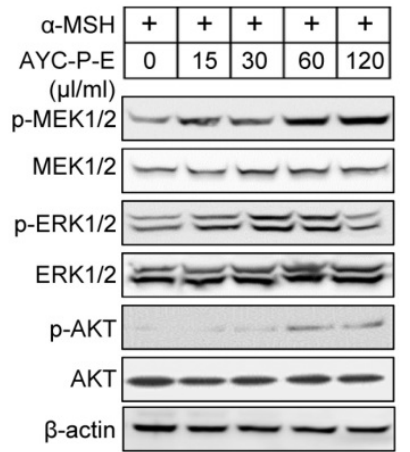

C

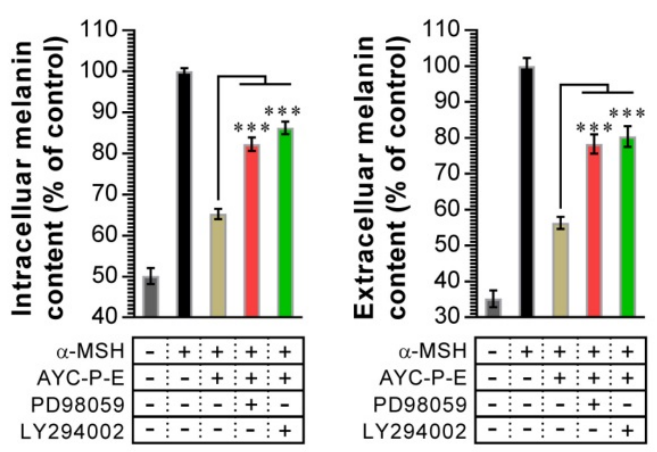

B
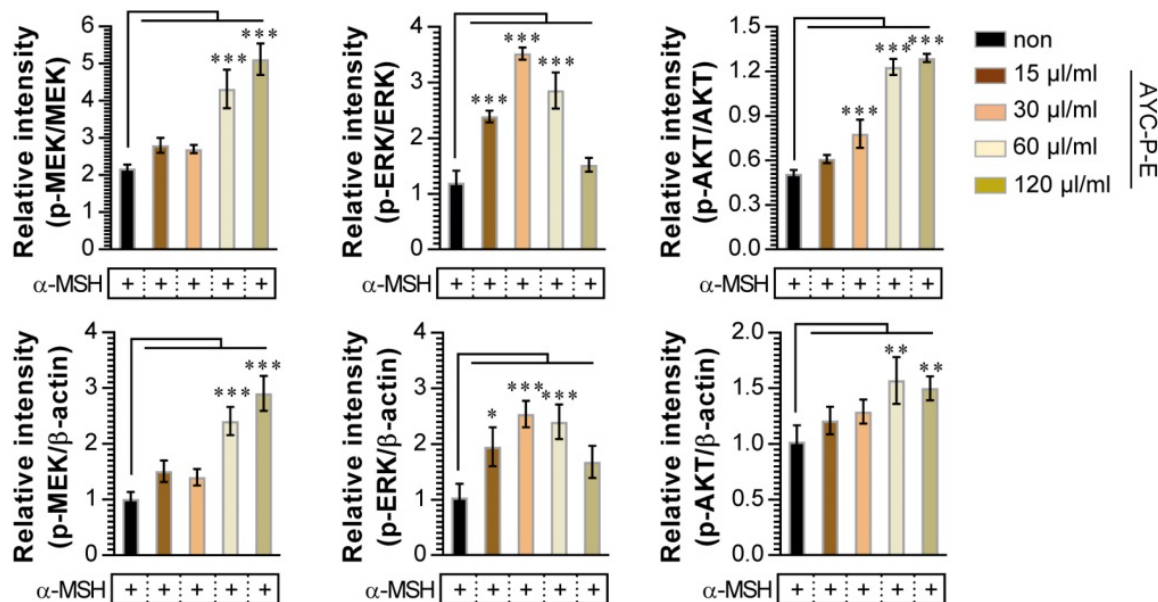

Figure 4. Effect of AYC-P-E treatment on MEK/ERK and AKT signals in $\alpha-M S H-s t i m u l a t e d ~ B 16 F 10$ cells. (A) B16F10 cells were incubated in medium containing $\alpha-M S H(200 \mathrm{nM})$ for $1 \mathrm{~h}$ and treated with the indicated concentrations of AYC-P-E for $24 \mathrm{~h}$. Immunoblot analyses were performed using Abs specific to $\mathrm{P}-\mathrm{MEK} 1 / 2$ (Ser $\left.{ }^{217} / \mathrm{Ser}^{221}\right)$, p-ERK 1/2 (Thr202/Tyr 204), and p-AKT (Ser 473). MEK1/2, ERK1/2, AKT, and $\beta$-actin antibodies were used as the protein loading control. (B; top panel) The relative intensity of each protein level compared with total loading controls (MEK1/2. ERK1/2, and AKT) is expressed as the mean in the bar graphs. (B; bottom panel) The relative intensity of each protein level compared with $\beta$-actin is expressed as the mean in the bar graphs. (C) B16F10 cells were incubated in medium containing $\alpha-M S H$ (200 nM) for $1 \mathrm{~h}$, and treated with AYC-P-E $(120 \mu \mathrm{l} / \mathrm{ml})$ in the absence and presence of ERK (PD98059; $10 \mu \mathrm{M})$ and AKT (LY294002; $10 \mu \mathrm{M})$ inhibitors for $72 \mathrm{~h}$. The intracellular and extracellular melanin contents were measured in the supernatant and pellets. All bar graphs show the means \pm standard deviation (SD) of three samples. One representative plot out of three independent experiments is shown; ${ }^{*} \mathrm{P}<0.05,{ }^{* *} \mathrm{P}<0.01$, or ${ }^{* * * *} \mathrm{P}<0.001$.

\subsection{Effect of AYC-P-E on skin pigmentation in a clinical setting.}

Based on the above in vitro results, the effectiveness of AYC-P-E in improving hyperpigmentation was examined in 22 subjects with hyperpigmentation in the facial area for 8 weeks after treatment with the test sample containing AYC-P-E and the control sample not containing AYC-P-E, and the change in the melanin index was analyzed. We found that the melanin index of the test group was significantly lower $(\mathrm{P}<0.05)$ than that of the control group 2 weeks after the initiation of AYC-P-E treatment and until the completion of the clinical study ( 8 weeks). The test group, treated with the test sample containing AYC-P-E, demonstrated 3.33, 7.06, and $8.68 \%$ hyperpigmentation improvement after 2,4 , and 8 weeks, respectively, whereas, the control group, treated with the control sample that did not contain AYCE, demonstrated $0.48,1.41$, and $2.5 \%$ hyperpigmentation improvement after 2, 4, and 8 weeks, respectively (Fig. 5). These results suggested that AYC-P-E could be used to improve skin pigmentation.

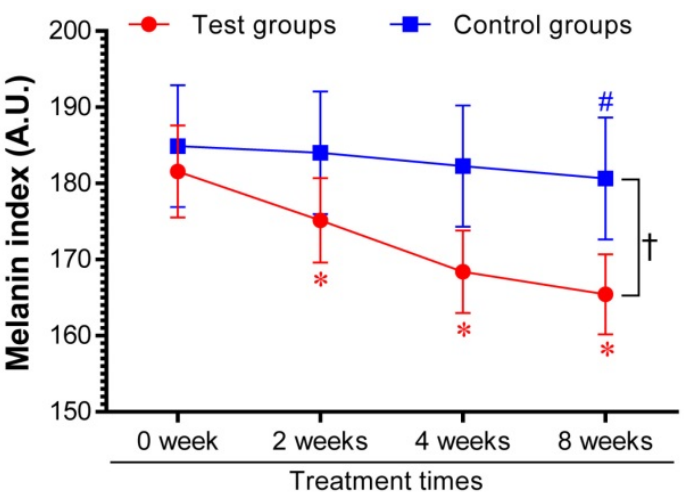

Figure 5. Changes in melanin index following product application for eight consecutive weeks. Twenty-two female patients with skin hyperpigmentation aged 37 to 55 participated in the study. The melanin index was analyzed at 2, 4, and 8 weeks after applying the test cream (Test groups) containing 68.6\% AYC-P-E, and the control cream (Control groups) not containing AYC-P-E. Data from one of two independent experiments are shown. Data represent the mean \pm standard error of measurement (SEM). ${ }^{*} \mathrm{P}<0.05$ compared to the melanin index before application $(0$ week) of the test cream containing AYC-P-E; $\# P<0.05$ compared to the melanin index before application ( 0 week) of the control cream not containing AYC-P-E; $+P<0.05$ comparison of the melanin index of the test and control groups at 2, 4 , and 8 weeks. 


\section{Discussion}

This study aimed to assess the effects of AYC-P-E extracted from an Aster yomena callus culture system on melanogenesis in vitro and in clinical settings. We observed that AYC-P-E reduced melanin synthesis via the activation of the ERK and AKT signaling pathways in a-MSH-stimulated B16F10 melanoma cells. In a clinical study in 22 patients with hyperpigmentation, AYC-P-E also significantly improved the skin melanin level.

Melanin biosynthesis is promoted as a mechanism against skin damage caused by exposure to UV rays [28]. Although melanin plays a positive role in protecting the skin, excessive melanin synthesis due to severe stimuli, such as UV rays may lead to pigmentation, such as freckles, and skin spots, and the toxicity of melanin precursors may lead to cell death and skin cancer [28, 29]. In fact, keratinocyte-derived mediators, such as a-MSH, basic fibroblastic growth factor, stem cell factor, endothelin-1, produced by UV stimulation can result in the activation of melanocytes, consequently promoting melanogenesis $[30,31]$. Interestingly, we recently observed that AYC-P-E exerts anti-photoaging functions in UVB-irradiated human keratinocytes by improving cell viability, inhibiting elastase, forming Type I procollagen, and inhibiting TNF-a. AYC-P-E is consists of 19 main metabolites including one isoflavonoid, one flavonoid, and three sphingolipids [22]. Among these, flavonoid compounds, such as isoflavonoids and flavonoids, have strong anti-oxidative, anti-carcinogenic, as well as anti-inflammatory effects [32, 33]. In particular, robustic acid is capable of inducing anti-tumor activity, while among sphingolipids, phytosphingosine plays an important role in the regeneration of damaged skin $[23,24]$. Although the anti-photoaging and whitening effects of each metabolite contained in AYC-P-E have not yet been identified, based on the above findings, the AYC-P-E-induced inhibition of melanin synthesis may be mediated by the presence of such metabolites.

Skin pigmentation is induced by melanogenesis, a complex process involving tyrosinases and other tyrosinase-related proteins (TRPs) in melanocytes [34]. Therefore, the mechanism of action of skin whitening agents is associated with reduced tyrosinase activity, which subsequently leads to the inhibition of melanogenesis [35]. Importantly, the expression of tyrosinase, TRP-1, and TRP-2 is induced by the activation of the CAMP/PKA/CREB signaling pathway which further increases MITF expression [36]. MITF is a master gene essential to the development and survival of melanocytes [37]. In this study, the anti-melanogenic activity of AYC-P-E induced the phosphorylation of CREB, which was accompanied by the a-MSH-induced inhibition of MITF in melanocytes. As a result, the mRNA and protein levels of tyrosinase, TRP-1 and TRP-2 decreased. Moreover, AYC-P-E treatment of a-MSH-stimulated B16F10 cells led to MEK/ERK and AKT phosphorylation. The MEK/ERK and AKT signaling pathways are closely related to MITF transactivation and stability [38]. For example, the activation of the MERK/ERK and AKT signaling pathways can promote the degradation of MITF in melanocytes that induce melanogenesis, thus resulting in anti-melanogenic activity [39, 40]. In fact, following the treatment of a-MSH-stimulated B16F10 cells with AYC-P-E and specific ERK and AKT inhibitors, the inhibitory effect of AYC-P-E on melanin synthesis was impaired. Therefore, the clinical effects of improved melanin levels in subjects with hyperpigmentation seem to be mediated by MITF degradation via the activation of ERK and AKT, accompanied by the subsequent inhibition of tyrosinase and other tyrosinase-related proteins.

In this study, inhibition of melanin synthesis by AYC-P-E was mediated by ERK and AKT activation, which resulted in improved melanin levels in patients with hyperpigmentation. This suggested the potential role of AYC-P-E as a functional cosmetic ingredient in skin whitening procedures or formulations. However, the whitening effects of each metabolite in AYC-P-E were not assessed. AYC-P-E contains a number of metabolites whose functionality has not been revealed. Therefore, further studies are required to identify the key metabolites of AYC-P-E possessing this whitening activity.

\section{Conclusions}

In conclusion, AYC-P-E extracted using a plant tissue culture technique induced MITF degradation followed by downregulation of melanin-inducing factors (tyrosinase, TRP-1 and TRP-2) in melanocytes that promote melanogenesis. Importantly, AYC-P-E inhibited melanogenesis by activating the ERK and AKT signaling pathways. In conclusion, our previous findings as well as the results of the present study on the anti-photoaging effects of AYC-P-E indicate that AYC-P-E may be used as an ingredient in functional cosmetic products for the treatment of various symptoms (including wrinkles, damaged skin barrier, hyperpigmentation, etc.) caused by photoaging.

\section{Abbreviations}

AYC-P-E: Aster yomena callus pellet extract; a-MSH: a-melanocyte stimulating hormone; CREB: cyclic AMP response element-binding protein; MITF: microphthalmia-associated transcription factor; 
TRP-1: tyrosinase related protein-1; TRP-2: tyrosinase related protein-2; MEK: mitogen-activated protein kinase; ERK: extracellular-signal-regulated kinase; AKT: protein kinase B; qRT-PCR: quantitative real-time polymerase chain reaction; PBS: Phosphate-buffered saline; ELISA: Enzyme-linked immunosorbent assay; SD: standard deviation; SEM: standard error of measurement.

\section{Acknowledgments}

This research was supported by This research was supported by the Ministry of Trade, Industry \& Energy (MOTIE), Korea Institute for Advancement of Technology (KIAT), industries of economic cooperation region, and the Korea Research Institute of Bioscience and Biotechnology (KRIBB) Research Initiative Program (KGM5242113 and KGM5282122).

\section{Author Contributions}

Conceptualization, W.S.K., Y-B.R.; Methodology, J-I.L., Y.J.J., C.Y.K., W.S.K.; Formal Analysis, J-I.L., E-S.K., S-M.C., J-H.J., J-R.K., J.H.S., J-D.C., W.S.K.; Investigation, J-I.L., Y-B.R., W.S.K.; Writing-Original Draft Preparation, W.S.K.; Writing-Review \& Editing, W.S.K.; Supervision, Y-B.R.; Project administration, Y-B.R. All authors have read and agreed to the published version of the manuscript.

\section{Ethics Committee Approval and Patient Consent}

The clinical research was approved (KDRI-IRB-20275) by the Institutional Review Board (IRB) of the Korea Dermatology Research Institute (KDRI). All protocols were initiated after receiving written consent in compliance with the principles of the Helsinki Declaration. The purpose and safety aspects of the study were explained to all patients and written informed consent was included in the documents of each patient.

\section{Competing Interests}

The authors have declared that no competing interest exists.

\section{References}

1. Klaschka U. Natural personal care products analysis of ingredient lists and legal situation. Environmental Sciences Europe. 2016; 28: 1-14.

2. Amberg N, Fogarassy C. Green consumer behavior in the cosmetics market. Resources. 2019; 8: 137 .

3. Bucheli TD, Strobel BW, Hansen HC. Personal care products are only one of many exposure routes of natural toxic substances to humans and the environment. Cosmetics. 2018; 5: 10.

4. Pereira L. Seaweeds as source of bioactive substances and skin care therapy Cosmeceuticals, algotheraphy, and thalassotherapy. Cosmetics. 2018; 5: 68.

5. Buchbauer G. On the biological properties of fragrance compounds and essential oils. Wiener medizinische Wochenschrift. 2004; 154: 539-47.

6. Acevedo-Whitehouse K, Duffus AL. Effects of environmental change on wildlife health. Philosophical transactions of the Royal Society of London Series B, Biological sciences. 2009; 364: 3429-38.
7. Trehan S, Michniak-Kohn B, Beri K. Plant stem cells in cosmetics: current trends and future directions. Future science OA. 2017; 3: FSO226.

8. Georgiev V, Slavov A, Vasileva I, Pavlov A. Plant cell culture as emerging technology for production of active cosmetic ingredients. Engineering in life sciences. 2018; 18: 779-98

9. Eibl R, Meier P, Stutz I, Schildberger D, Huhn T, Eibl D. Plant cell culture technology in the cosmetics and food industries: current state and future trends. Applied microbiology and biotechnology. 2018; 102: 8661-75.

10. Nugroho A, Kim MH, Choi J, Choi JS, Jung WT, Lee KT, et al. Phytochemical studies of the phenolic substances in Aster glehni extract and its sedative and anticonvulsant activity. Archives of pharmacal research. 2012; 35: 423-30.

11. Espinosa-Leal CA, Puente-Garza CA, Garcia-Lara S. In vitro plant tissue culture: means for production of biological active compounds. Planta. 2018; 248: 1-18.

12. Debnath SC, Arigundam U. In Vitro Propagation Strategies of Medicinally Important Berry Crop, Lingonberry (Vaccinium vitis-idaea L.). Agronomy. 2020; 10: 744 .

13. Hussain A, Qarshi IA, Nazir H, Ullah I. Plant tissue culture: current status and opportunities. Recent advances in plant in vitro culture. 2012: 1-28.

14. Karuppusamy S. A review on trends in production of secondary metabolites from higher plants by in vitro tissue, organ and cell cultures. Journal of Medicinal Plants Research. 2009; 3: 1222-39.

15. Zhong JJ. Plant cell culture for production of paclitaxel and other taxanes. Journal of bioscience and bioengineering. 2002; 94: 591-9.

16. Mendoza D, Arias JP, Cuaspud O, Arias M. Phytochemical Screening of Callus and Cell Suspensions Cultures of Thevetia peruviana. Brazilian Archives of Biology and Technology. 2020; 63.

17. Suman S. Plant tissue culture: A promising tool of quality material production with special reference to micropropagation of banana. Biochem Cell Arch. 2017; 17: 1-26.

18. Gallego-Jara J, Lozano-Terol G, Sola-Martínez RA, Cánovas-Díaz M, de Diego Puente T. A Compressive Review about Taxol®: History and Future Challenges. Molecules. 2020; 25: 5986.

19. Rhew Z-I, Lee JH, Han Y. Aster yomena has anti-arthritic activity against septic arthritis induced by Candida albicans: its terpenoid constituent is the most effective and has synergy with indomethacin. Advances in Traditional Medicine. 2019: 1-9.

20. Han MH, Jeong JS, Jeong JW, Choi SH, Kim SO, Hong SH, et al. Ethanol extracts of Aster yomena (Kitam.) Honda inhibit adipogenesis through the activation of the AMPK signaling pathway in 3T3-L1 preadipocytes. Drug discoveries \& therapeutics. 2017; 11: 281-7.

21. Hwang KA, Hwang YJ, Song J. Aster yomena extract ameliorates pro-inflammatory immune response by suppressing NF-kappaB activation in RAW 264.7 cells. Journal of the Chinese Medical Association : JCMA. 2018; 81: 102-10.

22. Kim WS, Seo JH, Lee JI, Ko ES, Cho SM, Kang JR, et al. The Metabolite Profile in Culture Supernatant of Aster yomena Callus and Its Anti-Photoaging Effect in Skin Cells Exposed to UVB. Plants. 2021; 10

23. Kim WS, Kim K, Byun EB, Song HY, Han JM, Park WY, et al. RM, a novel resveratrol derivative, attenuates inflammatory responses induced by lipopolysaccharide via selectively increasing the Tollip protein in macrophages: A partial mechanism with therapeutic potential in an inflammatory setting. International immunopharmacology. 2020; 78: 106072.

24. Ko GA, Kim Cho S. Ethyl linoleate inhibits alpha-MSH-induced melanogenesis through Akt/GSK3beta/beta-catenin signal pathway. The Korean journal of physiology \& pharmacology : official journal of the Korean Physiological Society and the Korean Society of Pharmacology. 2018; 22: 53-61.

25. Kim YM, Cho SE, Seo YK. The activation of melanogenesis by p-CREB and MITF signaling with extremely low-frequency electromagnetic fields on B16F10 melanoma. Life sciences. 2016; 162: 25-32.

26. Ma J, Guo W, Li C. Ubiquitination in melanoma pathogenesis and treatment. Cancer medicine. 2017; 6: 1362-77.

27. Khaled M, Larribere L, Bille K, Aberdam E, Ortonne JP, Ballotti R, et al. Glycogen synthase kinase 3beta is activated by cAMP and plays an active role in the regulation of melanogenesis. The Journal of biological chemistry. 2002; 277: 33690-7.

28. Brenner M, Hearing VJ. The protective role of melanin against UV damage in human skin. Photochemistry and photobiology. 2008; 84: 539-49.

29. Yang Z, Zeng B, Pan Y, Huang P, Wang C. Autophagy participates in isoliquiritigenin-induced melanin degradation in human epidermal keratinocytes through $\mathrm{PI} 3 \mathrm{~K} / \mathrm{AKT} / \mathrm{mTOR}$ signaling. Biomedicine \& pharmacotherapy = Biomedecine \& pharmacotherapie. 2018; 97: 248-54.

30. Jung JM, Noh TK, Jo SY, Kim SY, Song Y, Kim YH, et al. Guanine Deaminase in Human Epidermal Keratinocytes Contributes to Skin Pigmentation. Molecules. 2020; 25.

31. Gilchrest BA, Park HY, Eller MS, Yaar M. Mechanisms of ultraviolet light-induced pigmentation. Photochemistry and photobiology. 1996; 63: 1-10.

32. Sharma N, Tiwari V, Vats S, Kumari A, Chunduri V, Kaur S, et al. Evaluation of Anthocyanin Content, Antioxidant Potential and Antimicrobial Activity of Black, Purple and Blue Colored Wheat Flour and Wheat-Grass Juice against Common Human Pathogens. Molecules. 2020; 25.

33. Yu J, Bi X, Yu B, Chen D. Isoflavones: Anti-Inflammatory Benefit and Possible Caveats. Nutrients. 2016; 8

34. Jang DK, Pham $\mathrm{CH}$, Lee IS, Jung $\mathrm{SH}$, Jeong JH, Shin $\mathrm{HS}$, et al. Anti-Melanogenesis Activity of 6-O-Isobutyrylbritannilactone from Inula 
britannica on B16F10 Melanocytes and In Vivo Zebrafish Models. Molecules. 2020; 25.

35. Pillaiyar T, Manickam M, Namasivayam V. Skin whitening agents: medicinal chemistry perspective of tyrosinase inhibitors. Journal of enzyme inhibition and medicinal chemistry. 2017; 32: 403-25.

36. Qian W, Liu W, Zhu D, Cao Y, Tang A, Gong G, et al. Natural skin-whitening compounds for the treatment of melanogenesis (Review). Experimental and therapeutic medicine. 2020; 20: 173-85.

37. Levy C, Khaled M, Fisher DE. MITF: master regulator of melanocyte development and melanoma oncogene. Trends in molecular medicine. 2006; 12: 406-14.

38. Huang HC, Chang SJ, Wu CY, Ke HJ, Chang TM. [6]-Shogaol inhibits alpha-MSH-induced melanogenesis through the acceleration of ERK and PI3K/Akt-mediated MITF degradation. BioMed research international. 2014; 2014: 842569 .

39. Ha JH, Jeong YJ, Xuan SH, Lee JY, Park J, Park SN. Methyl-2-acetylamino-3-(4-hydroxyl-3,5-dimethoxybenzoylthio)propanoate suppresses melanogenesis through ERK signaling pathway mediated MITF proteasomal degradation. Journal of dermatological science. 2018.

40. Wang C, Zhao L, Su Q, Fan X, Wang Y, Gao S, et al. Phosphorylation of MITF by AKT affects its downstream targets and causes TP53-dependent cell senescence. The international journal of biochemistry \& cell biology. 2016; 80: $132-42$. 